\title{
Correction to: A space decomposition scheme for maximum eigenvalue functions and its applications
}

\author{
Ming Huang1,2 • Yue $\mathrm{Lu}^{3} \cdot \mathrm{Li}$ Ping Pang ${ }^{4}$. \\ Zun Quan Xia ${ }^{4}$
}

\section{Correction to: Math Meth Oper Res (2017) 85:453-490 https://doi.org/10.1007/s00186-017-0579-z}

A few mistakes were inadvertently overlooked in the following paper: https://doi.org/ 10.1007/s00186-017-0579-z. We apologize for the inconvenience caused.

Please find the corrections below:

1. Page 6, The signal '(2.6)' in Theorem 2.1 should be '(2.7)'.

2. Page 14, 'Line 10' and 'Line 14'. The formula ' $(v, G) \in w(u)\left[D A(x)^{\star}\right] \ldots$ '. should be ' $(v, G) \in w(u) \times\left[D A(x)^{\star}\right] \ldots$. .

3. Page 16, Line 15 . The formula ' $d \in \mathcal{U}_{f_{k}}\left(x^{*}\right)$ ' should be corrected as ' $d \in \mathcal{U}_{f}\left(x^{*}\right)$ '.

4. Page 25 , The last line. ' $k$ ' should be ' $k$ ' in the formula ' $M(0)$ '.

5. Page 26 , Line 7 . ' $k$ ' should be ' $k$ ' in ' $(k, l) \in I_{2}$ ' and ' $v_{k l}$ '.

6. Page 26, ' $W\left(u^{\mathbb{k}} ; 0\right)$ ' should be ' $w\left(u^{\mathbb{k}}\right)$ ' in the proof of Theorem 4.4.

7. Page 36, the forth line from the bottom. add '11701063' before 'the Project funded by ...'; the third line from the bottom. 'Fundamental Research Funds for the 13 Central Universities under Project No. 3132016108 ...' should be 'Fundamental 14 Research Funds for the Central Universities under Project No. 3132016108 and 3132017052....'.

The original article can be found online at https://doi.org/10.1007/s00186-017-0579-z.

Ming Huang

huangming0224@163.com

1 School of Control Science and Engineering, Dalian University of Technology, Dalian 116024, China

2 Department of Mathematics, Dalian Maritime University, Dalian 116026, China

3 School of Mathematical Sciences, Tianjin Normal University, Tianjin 300387, China

4 CORA, School of Mathematical Sciences, Dalian University of Technology, Dalian 116024, China 